\title{
Educação alimentar: promoção da saúde através da didatização nutricional associada à atividade física para diabéticos, hipertensos e \\ obesos
}

Nutrition education: health promotion though nutritional datialization associated with physical activity for a diabetes mellitus, hypertense end obese carriers

DÁrio Heider ViVeiros de Abre

Sueli de Souza Costa

\section{Resumo}

As doenças crônicas não transmissíveis como diabetis mellitus, hipertensão arterial sistêmica e obesidade estão associadas, por exemplo, a falta de atividade física regular e a ingestão de alimentos inadequados, caracterizando-se, portanto, como uma síndrome metabólica. Diante deste contexto estruturou-se como objetivo desse estudo intervir através da transposição didática nutricional associada a introdução de atividade física para a promoção da saúde dos portadores de tais comorbidades no município de Coelho Neto (MA), atendidos pela equipe oito e auxiliados pelo Núcleo de Apoio a Saúde da Família (NASF). Como metodologia para o desenvolvimento desse projeto de intervenção utilizou-se rodas de conversas, consultas médicas e uma pesquisa de campo com aplicação de um questionário com indagações fechadas sobre a interface da didatização nutricional e atividade física regular sobre a qualidade de vida dos pacientes assistidos. $\mathrm{O}$ resultado da intervenção observou uma relação direta dos pacientes mobilizados pelo projeto em sua qualidade de vida. O estudo concluiu que a perda de peso e a adoção de hábitos mais saudáveis foram as variáveis mais frequentes apontadas pelos pacientes, portanto, mais associadas a confecção desse cenário. Isso denotou uma perspectiva favorável para a diminuição de internações, frequência em urgências e emergências, dentre outras consequências associadas à carência de promoção da saúde em portadores das doenças crônicas evidenciadas nesta pesquisa.

Palavras-chave: Doenças Crônicas. Síndrome Metabólica. Transposição Didática. Nutricional. Atividade Física.

\footnotetext{
a Universidade Federal do Ceará (UFC), CE, Brasil. Especialista em Pesquisa e Inovação em Saúde da Família, e-mail: darioheider@hotmail.com https://orcid.org/0000-0003-4483-1001

${ }^{\mathrm{b}}$ Universidade Federal do Maranhão (UFMA), MA, Doutora em Ciências Odontológicas, Brasil. e-mail: scsueli@gmail.com, https://orcid.org/0000-0003-4127-7324

Rev. Caminhos da Educação: diálogos, culturas e diversidades, Teresina, v. 1, n. 3, p. 149-165, set./dez. 2019
} 


\begin{abstract}
Noncommunicable chronic diseases such as Diabetes Mellitus, Systemic Arterial Hypertension and Obesity are associated, for example, with a lack of regular physical activity and the ingestion of inadequate food, thus characterizing themselves as metabolic syndromes. Given this context, and with the assistance of team 008 and the Nucleus of Family Health Support (NASF), the objective of this study was to intervene and promote the health of patients with such comorbidities in the city of Coelho Neto (MA), through the means of investigating the nutritional didactic transposition associated with the introduction of physical activity. As a methodology for the development of this intervention project we used conversation wheels, medical consultations and a field research, with the help of a close-ended questionnaire, about the impact of nutritional education and regular physical activity on the quality of life of assisted patients. The results of the intervention observed a direct relationship between patients involved in the project and their subsequent quality of life. The study concluded that weight loss and the adoption of healthier habits were the most frequent variables mentioned by patients, and as such, the most pertinent to the execution of this scenario. This indicated a favorable outlook for the reduction of hospitalizations, emergencies and frequency of said emergencies, amongst other consequences associated with the lack of health promotion in patients with chronic diseases, as evidenced in this research.
\end{abstract}

Keywords: Chronic Diseases. Metabolic syndrome. Didactic Transposition. Nutritional Physical activity.

\title{
Resumen
}

Las enfermedades crónicas no transmisibles como la diabetes mellitus, la hipertensión arterial sistémica y la obesidad se asocian, por ejemplo, con la falta de actividad física regular y la ingestión de alimentos inapropiados, caracterizándose así como un síndrome metabólico. Dado este contexto, el objetivo de este estudio fue intervenir a través de la transposición didáctica nutricional asociada con la introducción de actividad física para promover la salud de las personas con tales comorbilidades en el la ciudad de Coelho Neto en la provincia de Maranhão ( Brasil), atendido por el equipo 008 y asistido por el Apoyo como médico de cabecera, o sea medico de la estratégia familiar (NASF). Como metodología para el desarrollo de este proyecto de intervención, se utilizaron círculos de conversación, consultas médicas e investigación de campo, con la aplicación de un cuestionario con preguntas cerradas sobre la interfaz de la enseñanza nutricional y la actividad física regular sobre la calidad de vida de los pacientes asistidos. El resultado de la intervención observó una relación directa entre los pacientes movilizados por el proyecto y su calidad de vida. El estudio concluyó que la pérdida de peso y la adopción de hábitos más saludables fueron las variables más frecuentes señaladas por los pacientes, por lo tanto, más asociadas con la creación de este escenario. Esto denotó una perspectiva favorable para la reducción de las hospitalizaciones, la frecuencia de urgencias y emergencias, entre otras consecuencias asociadas con la falta de promoción de la salud en pacientes con enfermedades crónicas evidenciadas en esta investigación.

Palabras clave: Enfermedades crónicas. Síndrome metabólico. Transposición didáctica. Nutricional. Actividad física.

Rev. Caminhos da Educação: diálogos, culturas e diversidades, Teresina, v. 1, n. 3, p. 149-165, set./dez. 2019 
Educação alimentar: promoção da saúde através da didatização nutricional associada à atividade física para portadores de diabetes mellitus, hipertensos e obesos

\section{INTRODUÇÃO}

Ao participar do Programa Mais Médicos fez-se notório o desconhecimento da maioria dos pacientes sobre o consumo de calorias, o mal-uso de alimentos e a falta da atividade física por mínima que seja. Esse fato acarreta inúmeros problemas de saúde, dentre outros, esteatose hepática, problemas osteomusculares e síndrome metabólica.

Este trabalho é o produto do intuito de intervir na educação alimentar, identificando os tipos de nutrição associados à atividade física mínima. Isto posto, formula-se o seguinte problema: Como a transposição didática nutricional, através da educação alimentar associada à introdução de atividade física, pode intervir com a promoção da saúde dos portadores de diabetes mellitus, hipertensos e obesos no município de Coelho Neto (MA), atendidos pela equipe número oito da Estratégia Saúde da Família (ESF) com o auxílio do Núcleo de Apoio a Saúde da Família (NASF).

A frequência expressiva de pacientes portadores de doenças crônicas atendidos pela equipe oito da Unidade Básica de Saúde (UBS) no município de Coelho Neto (MA) apresentou-se como uma realidade carente de um processo de intervenção, devido às implicações trazidas por essas enfermidades, principalmente na medida em que os pacientes não adotam hábitos saudáveis globais, de modo basilar, tais como: alimentação adequada e prática de atividade física regular.

A partir dessa demanda, entende-se que a intervenção em questão tornou-se relevante diante desse quadro comunitário tão afetado por essas problemáticas. Neste contexto, a intervenção ocorreu devido ao aumento de pacientes portadores de HAS, DM, síndrome metabólica, obesidade, e aumento de classes de medicamentos aos portadores de DM, HAS sem o controle do peso; aumento de pacientes com sobrepeso e obesidade associado à polimedicação, desconhecimento dos tipos de alimentos como carboidratos, proteínas e gorduras, aumento do consumo de alimentos industrializados e pobres em nutrientes associado a não prática da atividade física e, por vezes, a precariedade das condições de orientação nutricional nas UBS.

Este trabalho tem como objetivo geral refletir sobre a reeducação alimentar através da transposição didática nutricional, associada a introdução de atividade física, para a promoção da saúde dos portadores de diabetes mellitus e hipertensos no município de Coelho Neto (MA), atendidos pela equipe número oito e auxiliado pelo Núcleo de Apoio a Saúde da Família (NASF).

Rev. Caminhos da Educação: diálogos, culturas e diversidades, Teresina, v. 1, n. 3, p. 149-165, set./dez. 2019 
Esse projeto de intervenção consistiu em um processo de articulação e mobilização junto aos pacientes atendidos por uma equipe do Núcleo de Apoio a Saúde da Família (NASF). Dessa forma constituiu-se as seguintes etapas para a construção dessa intervenção: A Criação de cartilha para orientação alimentar, associado à introdução de uma atividade para pacientes polimedicamentados e acompanhados pela equipe do NASF.

Nesta intervenção, buscou-se a criação de uma cartilha de fácil orientação e compreensão para a identificação de alimentos, acompanhamentos com equipe do NASF, conscientização da necessidade da atividade física através de rodas de conversas (grupo focal), visitas domiciliares e consultas.

O estudo foi realizado entre os meses de junho a novembro de 2019, na cidade de Coelho Neto (MA), mais especificamente na Unidade Básica de Saúde São Francisco na qual atua a equipe oito da Estratégia Saúde da família. A amostra deteve-se aos pacientes hipertensos, diabéticos, com síndrome metabólica e obesos atendidos pela supracitada equipe.

As mudanças sociais, econômicas e demográficas através da transição epidemiológica no Brasil contribuíram para o acréscimo considerável da morbimortalidade por Doenças Crônicas Não Transmissíveis (DCNT), nas quais a Hipertensão Arterial Sistêmica (HAS) e o Diabetes Mellitus (DM) estão entre as mais frequentes. $\mathrm{O}$ enfrentamento destas doenças configura-se em um desafio para a saúde pública, considerando que ambas têm um forte impacto na vida dos indivíduos afetados, causado morte prematura e gerando grandes e subestimados efeitos econômicos adversos para as famílias, comunidades e sociedade em geral (BRASIL, 2005).

No Brasil, a implantação do Sistema Único de Saúde (SUS) na década de 1990 representou uma importante mudança no padrão de organização dos serviços de saúde no país, sobretudo com o fortalecimento da atenção básica de saúde. Com a implantação do Programa de Saúde da Família (PSF), foi estabelecido um direcionamento geográfico da atenção por meio de equipes de saúde da família nas áreas e subgrupos populacionais desfavorecidos socialmente. A Estratégia de Saúde da Família (ESF) é, atualmente, considerada como a principal porta de entrada ao sistema de saúde. Entre as principais responsabilidades da atenção básica estão o controle da hipertensão e do diabetes (BRASIL, 2005).

A utilização dos substratos energéticos necessários para manutenção de uma vida saudável a partir de uma alimentação adequada, e as necessárias quantidades a serem Rev. Caminhos da Educação: diálogos, culturas e diversidades, Teresina, v. 1, n. 3, p. 149-165, set./dez. 2019 
Educação alimentar: promoção da saúde através da didatização nutricional associada à atividade física para portadores de diabetes mellitus, hipertensos e obesos

consumidas são fundamentais para a manutenção da vida basal. Para os estudiosos de alimentos, o conhecimento de cada macro e micronutriente necessário para uma vida basal e uma vida associada a uma atividade física, seja ela uma rotina de caminhada três vezes por semana, uma corrida diária, um atleta amador de academia para a manutenção de um corpo atlético ou mesmo a um atleta de alto desempenho, seja ele (a) um bodybuilding ou atleta olímpico são fatores elementares para a promoção da saúde de cada indivíduo (COSTA; MARQUEZI, 2008).

Sendo assim, saber como a energia fornecida pelos alimentos é utilizada e armazenada se torna obrigação de quem se propõe a auxiliar pessoas ao ganho ou a eliminação de peso, seja perda de massa gorda ou mesmo a ganha de musculatura magra associado a uma dieta pertinente, com o consumo adequado às individualidades. (COSTA; MARQUEZI, 2008).

Segundo George Herbert (1593-1633), qualquer que seja o pai da doença, uma má alimentação foi a mãe. Desta maneira, é indispensável, não por um mero modismo da utilização do termo dieta, a utilização da mesma para o controle da ingestão racional das inúmeras substâncias que deglutimos pela boca. Desta forma, Médart (2005) menciona Larousse, que define dieta como um "conjunto de regras ou de prescrições médicas que dizem respeito à alimentação e que se destinam a manter ou a restabelecer a saúde" (p. 19).

Assim, existe uma dieta ideal que chamamos de alimentação ótima, a mesma é construída em função do conjunto de critérios nutricionais conhecidos pelas suas propriedades susceptíveis de manter ou de restabelecer a saúde (LAROUSSE, 2015). Esta alimentação ótima será moldável de acordo com as necessidades próprias de cada pessoa ou em função de suas circunstâncias particulares, sejam elas relacionadas à gestação, como também, ao sobrepeso, obesidade, diabetes, hipertensão arterial sistêmica, problemas digestivos, desnutrição ou mesmo à suplementação para atletas ou praticantes de atividade atlética amadora.

Segundo Médart (2015), a cada dia se ingere cada vez mais açúcares como a sacarose e amidos refinados, multiplicando-se assim, a ingestão de calorias que se chamam de ocas, vazias, pobres em micronutrientes. O consumo de glicídios complexos ou sem refinamento tais como lentilhas, feijões, grão-de-bico dentre outros, têm diminuído a cada dia, o consumo de proteínas vegetais também tem sido reduzido. Ingerem-se cada dia mais matérias gordas, e essencialmente sob forma de gorduras

Rev. Caminhos da Educação: diálogos, culturas e diversidades, Teresina, v. 1, n. 3, p. 149-165, set./dez. 2019 
escondidas. De acordo com este autor, o relatório dos trabalhos da Internacional Task Force, publicado em 1998 nos EUA, um em cada cinco americanos pesa mais de $100 \mathrm{~kg}$ e $70 \%$ da população apresenta um excesso de peso.

Desta maneira, e a partir das informações sobre consumo exagerado de produtos vazios em micronutrientes e o aumento de peso, nota-se que pelos motivos das facilidades de consumo de algumas populações, tais pessoas com sobrepeso e a falta de atividade física, tem desenvolvido diversas patologias que afetam sistemicamente as funções e órgão do corpo humano. É notório encontrar no consultório pessoas sedentárias referindo cansaço, dores articulares, vertigens, fraqueza muscular, além de elevação dos colesteróis e da glicemia (MENOR; SANTOS, 2012).

Portanto, é de responsabilidade do profissional de saúde o papel de educador, fazer os pacientes compreenderem que a maioria dos sinais e sintomas está relacionada a uma dieta inadequada e a falta de uma atividade física. Pode-se enfatizar que tal atividade seja leve a princípio e que traga determinado prazer para quem se propões a realizá-la (MENOR; SANTOS, 2012).

$\mathrm{Na}$ medida em que se envelhece e engorda-se inadequadamente ocorre um processo de lipossubstituição, que é a redução da musculatura em consequência do aumento da gordura corporal. Esse processo de redução da musculatura pode apresentar quadro de astenia evoluindo para cansaço e o aumento da pressão arterial, visto que o organismo entende que há um grande volume a ser perfundido para a manutenção daquele corpo excessivamente grande, com possível surgimento de dislipidemias (BRASIL, 2005).

\section{TRANSPOSIÇÃO DIDÁTICA E DIDATIZAÇÃO}

[...] desafio é arrimar e articular os saberes acadêmico aos saberes práticos ou da experiência profissional. Aliás, o grande desafio da formação inicial hoje é abolir o modelo acadêmico disciplinar que está na base da tradição universitária. Reagrupando, por exemplo, os saberes e os conhecimentos necessários ao profissional em função de outras bases que a disciplinar, quais sejam: saberes e competências para agir em classe (o saber agir, pilotar, supervisionar as ações em classe), saberes e competências necessários para planejar, organizar e elaborar uma lição (os conhecimentos disciplinares, o saber conceber, elaborar, planejar situações de ensino-aprendizagem), ou ainda, saberes e competências para compreender e agir em diferentes contextos profissionais (a história e os fundamentos da profissão, a escola,

Rev. Caminhos da Educação: diálogos, culturas e diversidades, Teresina, v. 1, n. 3, p. 149-165, set./dez. 2019 
Educação alimentar: promoção da saúde através da didatização nutricional associada à atividade física para portadores de diabetes mellitus, hipertensos e obesos

a clientela, a profissionalidade, etc.) e isso de forma a colocar estes diferentes saberes (saberes declarativos, saberes processuais ou semiprocessuais, etc.) a serviço da prática do futuro docente (Borges, 2009, p. 67).

A epígrafe de Borges (2009) refere-se ao conjunto de saberes necessários para o desempenho do professor, da docência, portanto. Entretanto, na área de saúde e, de modo particular nos processos de intervenção das equipes de saúde junto as populações atendidas esse processo de sistematização é extremamente necessário.

É fundamental para o diálogo com pacientes diversos a noção dos tipos de saberes que vão ser utilizados e considerados no processo de comunicação, da transposição didática e da didatização. Isto posto, faz-se necessário apresentar as tipologias de saberes consideradas pela literatura, com a finalidade de promover a relação com os conceitos de transposição didática e didatização.

A sistematização dos saberes tornou-se uma inquietação no bojo das Ciências da Educação, culminando em produções, implicando em sua tipologia. Diversos autores nas últimas três décadas, a partir de pontos de vista teóricos diferentes, propõem especificar tipologias dos saberes docentes.

De certo modo, esta multiplicidade de pesquisas expande a temática sobre os "saberes". Sem ter a pretensão de dissipar seus conceitos, analisa-se algumas propostas, apresentando autores que demarcaram este processo de sistematização a partir dos anos de 1980 elaborando uma interrelação com o bojo estrutural de saberes constituídos por Tardif (2009).

O conhecimento do conteúdo, segundo Shulman (1986), aborda exclusivamente a matéria pertinente ao docente, relacionada e concordante com sua área de conhecimento. É o domínio do conteúdo da disciplina que será dispersada através da ação docente. Conhecer o conteúdo, segundo este autor, remete o docente a desenvolver as habilidades de explicar e contextualizar a disciplina de forma que sejam concebidas à realidade do discente, ajudando a motivá-los no processo de aprendizado:

Teachers must not only be capable of defining for students the accepted truths in a domain. They must also be able to explain why a particular proposition is deemed warranted, why it is worth knowing, and how' it relates to other propositions, both within the

Rev. Caminhos da Educação: diálogos, culturas e diversidades, Teresina, v. 1, n. 3, p. 149-165, set./dez. 2019 
discipline and without, both in theory and in practice.

1. (Shulman: 1986, p. 9)

Pimenta (1995) sugere que os "Saberes do Conhecimento" refere-se ao conteúdo a ser ministrado e são adquiridos através do processo de formação acadêmica. Este saber, portanto, é ligado à disciplina na qual o docente atua.

Os saberes disciplinares foram abordados de uma forma tácita, contidos ou associados a outros parâmetros de classificação pelos demais autores estudados, como por exemplo, Malglaive (1990), que o inclui como um dos "Saberes Teóricos".

Tamir (1991) refere-se aos saberes disciplinares como sendo um dos componentes dos "Saberes Formais". Por Nóvoa (1992), que o classifica como um dos eixos do "Saber", que se diferencia dos outros eixos "Saber-fazer" e "Saber-ser". E por Barbier (1996), que trata os como um dos "Saberes Objetivados".

Para Malglaive (1990), saber teórico não revela o que se tem que fazer, mas conduz a atuar com discernimento, construindo procedimentos rigorosos e dando os meios de identificar as prováveis consequências das ações docentes em determinadas condições (MALGLAIVE, 1990).

Os saberes curriculares propostos por Tardif (2009) referem-se à maneira como as instituições de ensino gerenciam os conhecimentos socialmente produzidos e que devem ser transmitidos aos discentes. Materializam-se através dos objetivos, conteúdos e métodos dos programas escolares, que os docentes devem aprender, dominar e aplicar.

O saber curricular, abordado por Shulman (1986) como "Conhecimento Curricular" norteia o docente no sentido do domínio estruturante da disciplina que irá atuar, como por exemplo, os níveis dos conteúdos, a disposição do programa, os currículos disciplinares. Cabe ao docente a compreensão desta estrutura para a elaboração do que será ministrado, contextualizado a quem será ensinado. Neste sentido, Shulman (1986) frisa a necessidade de compreender simultaneamente dois tipos de currículos, o currículo lateral ("lateral curriculum”) e o currículo vertical ("vertical curriculum”), desta forma expressos:

This lateral curriculum knowledge [...] underlies the teacher's ability to relate the content of a given course or lesson to topics

\footnotetext{
1 Tradução livre: “Os professores não devem apenas ser capazes de definir para os alunos as verdades aceitas em uma área. Eles também devem ser capazes de explicar por que uma proposição em particular é considerada justificável, por que vale a pena conhecê-la e como ela se relaciona com outras proposições, tanto dentro quanto fora da disciplina, tanto na teoria quanto na prática”.

Rev. Caminhos da Educação: diálogos, culturas e diversidades, Teresina, v. 1, n. 3, p. 149-165, set./dez. 2019
} 
or issues being discussed simultaneously in other classes. The vertical equivalent of that curriculum knowledge is familiarity with the topics and issues that have been and will be taught in the same subject area during the preceding and later years in school, and the materials that embody them ${ }^{2}$. (SHULMAN, 1986, p. 10)

Os verbos utilizados por Shulman (1986) indicam a necessidade de o professor relacionar o conhecimento de um determinado conteúdo com o processo de discussão presente sobre o tema, neste sentido constituído de conhecimento do currículo lateral. Outro verbo trata a capacidade de abordar as familiaridades dos conteúdos presentes com assuntos que já foram dirimidos ou que ainda serão constituídos.

Através da categoria "Saberes Contextuais" (“context knowledge”) Grossman (1988) contempla os saberes curriculares propostos por Tardif (2009). Os saberes contextuais referem-se a compreensão do contexto local e regional da instituição de ensino e da sala de aula, ou seja, sua inserção no espaço, na comunidade, aportando um conhecimento sobre o contexto social, cultural e político.

Um entendimento mais amplo a respeito dos discentes e sobre como a evolução da comunidade influencia a atuação docente. Semelhante proposta a estes saberes é elaborada por Carlsen (1991), em categorização denominada "Saberes sobre o Contexto Educativo Geral".

Na prática médica umas das formas de prevenir doenças é através do diálogo com o paciente ou com a comunidade, por exemplo. Entretanto, é necessário constituir estratégias para que o repasse de informações seja efetuado com êxito e, consequentemente promova os resultados esperados.

Em populações do interior do Brasil esse contexto pode ser mais complexo, devido aos baixos níveis de escolarização dos pacientes e, principalmente por questões culturais que imprimem hábitos que em muitos casos contrariam as questões de saúde individual e coletiva.

Esse contexto é muito comum nas equipes que trabalham com a saúde da família nas periferias das grandes cidades e no interior dos Estados do Brasil exigindo, portanto, a construção de estratégias para imprimir ações que definitivamente funcionem e se adéqüem a realidade das pessoas assistidas.

\footnotetext{
2 Tradução livre: "O conhecimento do currículo lateral [...] fundamenta a capacidade do docente de relacionar o conteúdo de um determinado curso ou lição a tópicos ou assuntos que estão sendo discutidos simultaneamente em outras turmas. O equivalente vertical desse conhecimento curricular aborda a familiaridade com os tópicos e questões que foram e serão ensinados na mesma área de assunto durante os anos anteriores e posteriores, e os respectivos materiais que os incorporam".

Rev. Caminhos da Educação: diálogos, culturas e diversidades, Teresina, v. 1, n. 3, p. 149-165, set./dez. 2019
} 
A partir dessa demanda estrutura-se nessa seção a apresentação de dois conceitos fundamentais para a estruturação dessa pesquisa, a saber: transposição didática e didatização. É importante salientar que essas ideias partem da Pedagogia e, podem ser devidamente adaptadas a realidade das necessidades de "ensino" ou intervenção efetuadas pelos profissionais das equipes de saúde da família.

Isto posto, busca-se estruturar uma interface desses relevantes conceitos com a conjuntura vivenciada no NASF de Coelho Neto (MA) em relação ao processo de intervenção articulado para os pacientes com doenças crônicas não transmissíveis.

Introduzidos esses conceitos, portanto, e, de suma elucidação materializa-se o diálogo com a informação médica no processo entendido como de "ensino e aprendizagem" dos pacientes e, nessa perspectiva, auxiliando no contexto de transformação dessas pessoas em prol de sua saúde e da comunidade aonde habitam.

O conceito de Transposição Didática (TD) foi primeiramente apresentado por o sociólogo Michel Verret, em 1975, na França, aonde cunha o termo Transposição didática (GOMES e MENDES, 2017), nesse sentido a TD seria de acordo com esses autores:

Nessa esteira, a TD pressupõe a existência de um processo, e que um conteúdo do conhecimento, tendo sido designado como saber a ser ensinado sofre, a partir daí, um conjunto de transformações adaptativas que o levam a tomar lugar entre os objetos de ensino. Assim, a TD é o percurso de transformações ocorridas no saber de referência até se tornar um saber da sala de aula (p. 321).

Nessa mesma perspectiva Dominguini (2008, p. 2) contribui com o conceito de Transposição Didática dessa forma compreendido:

o conhecimento científico é organizado na forma de conteúdos escolares, didaticamente elaborados para permitir sua transmissão por parte do professor e uma possível assimilação por parte dos alunos. Os conteúdos são um conjunto de saberes que o contexto social vigente compreende como necessário a ser transmitido às novas gerações.

A Transposição Didática, entende-se que ela configura-se como um relevante processo de ensino e aprendizagem, que sofre constantes mudanças, em decorrência das possibilidades das dinâmicas sociais, econômicas, culturais, dentre outras constituindose, assim, que o saber científico torne-se ensinável no espaço escolar ou em qualquer outra dimensão que se ocupe com a propagação desse conhecimento.

Rev. Caminhos da Educação: diálogos, culturas e diversidades, Teresina, v. 1, n. 3, p. 149-165, set./dez. 2019 
A TD, portanto, passaria por fases, desse modo descrita na figura 1:

Figura 1: Processo de Transposição Didática

1) CONHECIMENTO CIENTÍFICO SOBRE DETERMINADO SABER

a) Construção do conceito científico

2) CONHECIMENTO A SER ENSINADO

b) A entrada dos saber científico no currículo caracterizando o saber como objeto de ensino

3) CONHECIMENTO SOBRE O SABER EFETIVAMENTE ENSINADO

c) A retratação do saber nos livros didáticos, materiais paradidáticos e/ou dispositivos e modos de textualização escolar do saber científico

\section{3) CONHECIMENTO SOBRE O SABER EFETIVAMENTE APRENDIDO}

d) Do saber de ensino, caracterizado como objeto de ensino, até a sua assimilação pelo estudante.

Fonte: Chevellard (1985), adaptado de Gomes e Mendes (2017, p.322).

A figura 1 mostra o processo de transposição didática no contexto escolar, entretanto, é necessário expressar alguns esclarecimentos em relação a adaptação dessa conjuntura (processo de transposição didáticão ao ambiente de uma ação de intervenção na área de saúde em uma UBS - Unidade Básica de Saúde.

O Conhecimento a ser ensinado disposto na fase 1 , refere-se a prática de atividade física regular e uma nutrição promotora da saúde com a finalidade de auxiliar no tratamento dos pacientes com doenças crônicas não transmissíveis oriundos do conhecimento científico dos profissionais do NASF. O material utilizado refere-se a cartilha nutricional e as aulas de zumba promovidas pelo educador físico (processo a ser esclarecido na metodologia). O conhecimento sobre o efetivamente aprendido encontrase nas anamneses efetuadas pelo médico do programa ao longo do processo de intervenção, tendo como sujeito não o estudante, mas o paciente assistido pela UBS.

Rev. Caminhos da Educação: diálogos, culturas e diversidades, Teresina, v. 1, n. 3, p. 149-165, set./dez. 2019 
Nesse intervalo é importante questionar como levar o conhecimento científico para o ambiente das populações assistidas pelo UBS. É a partir desse contexto que a elaboração de materiais e atividades podem propiciar a facilitação dessa ação em um cenário repleto de óbices devido a estrutura e características dessa população.

Esse cenário denota o contexto de um município em situação de pobreza e, portanto, apresentando características que exigem da equipe no NASF maior sensibilidade para promover a propagação da informação e consequentemente imprimir um processo de instrução e educação para a saúde deste contingente, sendo considerado os saberes a implicar numa transposição didática para imprimi-los.

A transposição didática seria uma filosofia adequada para direcionar adequadamente o processo de intervenção nesse contexto. Numa perspectiva simples a TD é a capacidade de tornar o saber sábio (o saber erudito desenvolvido por cientistas) em um saber capaz de ser ensinado no espaço escolar e, no campo, ora apresentado, a escola seria o espaço do NASF, necessitando, portanto, de transformações e adequações. Nesse sentido Martiny e Silva (2014, p. 1) comentam:

Um dos grandes enfrentamentos que vem sendo realizado tem acontecido na perspectiva de estabelecer quais saberes, dentro de um universo expressivo de conhecimentos, habilidades e competências, necessitam ser abordados nos diferentes contextos $[\ldots]$.

Desse modo, estruturou-se a seguinte figura 2 adaptada a sistematização da articulação desenvolvida com o NASF e equipe número oito do município de Coelho Neto fundamentado na transposição didática.

Figura 2: Processo de Transposição Didática

1) CONHECIMENTO CIENTÍFICO SOBRE DETERMINADO SABER

a) Construção do conceito científico: acadêmico e devidamente apropriado pela equipe médica do NASF Coelho Neto.

\section{2) CONHECIMENTO A SER ENSINADO}

b) A entrada do saber científico no currículo caracterizando o saber como objeto de ensino: agregar aos protocolos de atendimento do NASF difundido através do processo de intervenção.

Rev. Caminhos da Educação: diálogos, culturas e diversidades, Teresina, v. 1, n. 3, p. 149-165, set./dez. 2019 


\section{3) CONHECIMENTO SOBRE O SABER EFETIVAMENTE ENSINADO}

c) A retratação do saber nos livros didáticos, materiais paradidáticos e/ou dispositivos e modos de textualização escolar do saber científico: materiais como a cartilha nutricional e as aulas de zumba levando em conta os saberes relacionados a ciência e aos saberes do paciente.

\section{3) CONHECIMENTO SOBRE O SABER EFETIVAMENTE APRENDIDO}

d) Do saber de ensino, caracterizado como objeto de ensino, até a sua assimilação pelo estudante: pesquisa efetuada pelo médico através da anamnese relacionando com aspectos da qualidade de vida do paciente, verificando sua devida repercussão aos que aderiram.

Fonte: Chevellard (1985), adaptado pelo autor (2020).

Nesse sentido utiliza-se as palavras de Agranionih (2001) que afirma que o aprender e o ensinar são processos próprios dos seres humanos e acontecem ao longo da vida em contextos informais, como familiares, na natureza, na comunidade, no cultivo das tradições culturais e de valores, em contexto formais como a escola e no contato com os conhecimentos clássicos construídos ao longo da história da humanidade.

A didatização, portanto, assume liderança nesse processo de intervenção constituída dos saber científico (saber sábio) difundido através da eleição de um conteúdo (material e métodos) para a promoção do conhecimento. Nesse contexto, Pigatto e Bisognin (2017, p. 322) esclarecem:

Percebe-se, pois, que para ter êxito nos três níveis, o professor necessita passar por duas fases fundamentais, que se constituem como transposição externa e interna. A fase de Transposição Didática externa ocorre entre o saber sábio e o saber ensinar, e caracteriza-se pela análise dos conteúdos programáticos. E a fase de Transposição Didática interna, configura-se junto ao planejamento do currículo formal e pela decodificação do saber científico para uma linguagem que seja acessível aos estudantes, portanto esta fase se dá entre o saber ensinar e o saber ensinado.

A citação demonstra claramente a necessidade de utilização de mecanismos que efetivamente contribuíam com os diálogos e promova a apropriação da informação, do

Rev. Caminhos da Educação: diálogos, culturas e diversidades, Teresina, v. 1, n. 3, p. 149-165, set./dez. 2019 
conhecimento por parte dos interlocutores. Nessa perspectiva a equipe médica (professores) e os pacientes participantes do processo de intervenção entendidos como alunos.

A produção da cartilha apresentou como fundamento a linguagem local e a oferta de alimentos disponíveis na comunidade. A aula de zumba foi apontada como processo de intervenção por apresentar coreografias de fácil assimilação, considerando a diversidade de pacientes (faixa etária e com comorbidades que incapacitam individualmente cada pessoa). As reuniões e visitas constituídas nesse processo também atendeu ao contexto dessa filosofia, nesse caso os profissionais interagiam de acordo com a linguagem dos locais e suas demandas.

A continuação do Programa "Mais Médico" no ano de 2018 no município de Coelho Neto (MA) trouxe o convício diário no consultório com pacientes portadores de doenças crônicas não transmissíveis sugerindo a necessidade de intervenção da equipe do NASF número oito que atende bairros da periferia desse município.

A partir da verificação de um número expressivo de pacientes com diabetes mellitus, hipertensos e obesos, enfermidades provocadas principalmente por uma alimentação inadequada e ausência de atividade física, a equipe do NASF número oito (constituída por enfermeiro, técnico de enfermagem, agente de saúde comunitário, nutricionista, educador físico, fisioterapeuta, psicólogo, assistente social e médico) resolveu criar um processo de intervenção com o propósito de imprimir uma mudança de rotina para promover a saúde desses pacientes e verificar como a mudança de cotidiano, através de hábitos alimentares mais saudáveis e prática de atividade física regular, poderiam implicar na melhoria da qualidade de vida desses pacientes.

A partir dessa demanda criou-se um cronograma para promover um processo de articulação e mobilização junto aos pacientes portadores das doenças crônicas não transmissíveis mais frequentes constituindo um processo de intervenção com duas ações, foram elas a distribuição de uma cartilha com sugestão de hábitos alimentares mais saudáveis (elaborada pela nutricionista e o médico) e a inserção de atividade física através do educador físico com aulas de zumba (dança) três vezes por semana, além da recomendação de caminhadas de no mínimo trinta minutos três vezes por semana.

Diante desse processo de mobilização, foi indagado a esses sujeitos sobre a relação da intervenção realizada com a melhoria nos aspectos associados pela Organização Mundial de Saúde (ALMEIDA; GUTIERREZ; MARQUEZ, 2012, p. 1) como qualidade de vida, ou seja, "a percepção do indivíduo de sua inserção na vida, no Rev. Caminhos da Educação: diálogos, culturas e diversidades, Teresina, v. 1, n. 3, p. 149-165, set./dez. 2019 
Educação alimentar: promoção da saúde através da didatização nutricional associada à atividade física para portadores de diabetes mellitus, hipertensos e obesos

contexto da cultura e sistemas de valores nos quais ele vive e em relação aos seus objetivos, expectativas, padrões e preocupações". Nesse contexto lista-se o bem-estar espiritual, físico, mental, psicológico e emocional, além de relacionamentos sociais, como família e amigos e, também, saúde, educação, habitação saneamento básico e outras circunstâncias da vida. A partir desse conceito sistêmico sobre qualidade de vida abordouse aos pacientes após o início da intervenção da equipe do NASF os seguintes aspectos tais como: Perda de peso, Aumento da disposição geral, Aumento da disposição no trabalho ou atividade profissional, Melhoria nos relacionamentos pessoais, Melhoria da autoestima, Adoção de hábitos mais saudáveis, Ampliação do lazer, Diminuição das taxas relacionadas à HAS, DM e obesidade e Diminuição do stress.

Isto posto, diante dessas possibilidades ao longo do período de seis meses, de junho a novembro do ano de 2019, dos 753 pacientes que sofreram o processo de intervenção da equipe do NASF, 483 mobilizaram-se diante dessa abordagem. Foi considerado mobilizado ou incluído como participante do processo de intervenção os pacientes que compareceram ao ambulatório do posto de saúde pelo menos 2 vezes no período de junho a novembro. A relação das atividades propostas com a qualidade de vida foi questionada nas consultas no posto de saúde, além das visitas domiciliares e rodas de conversas efetuadas pela equipe.

\section{CONSIDERAÇÕES}

O processo de intervenção efetuado pela equipe oito do município de Coelho Neto (MA) trouxe para 483 pessoas mudanças importantes em suas rotinas associadas aos hábitos alimentares e a atividade física, portanto se relacionando diretamente com a qualidade de vida desses indivíduos.

Nesse sentido é relevante ressaltar que as transformações na rotina dos pacientes devem ser acompanhadas diretamente pelos profissionais de saúde envolvidos nesse processo, sendo que o principal propósito da equipe é mobilizar e promover efetivamente a educação para a saúde desses sujeitos.

Ocorre uma necessidade de efetuação dessas intervenções já que a partir da expressão de novos contextos diminui-se a mortalidade, sequelas, o número de visitas domiciliares, a demanda por internações hospitalares, diminuição do número de consultas de emergência e urgência provocadas por doenças crônicas não transmissíveis e a falta de um processo de intervenção preventivo e promotor da saúde desses pacientes.

Rev. Caminhos da Educação: diálogos, culturas e diversidades, Teresina, v. 1, n. 3, p. 149-165, set./dez. 2019 
É necessário, entretanto, que a adoção desse processo de intervenção seja permanente, continuo e global para que a saúde pública do município assistido amplie a qualidade de vida de seus habitantes saindo de um contexto curativo para uma conjuntura promotora da saúde. O resultado da intervenção demonstrou que uma mudança de rotina pode provocar na qualidade de vida das pessoas, expressando-se, portanto, como um importante mecanismo de política pública de saúde.

\section{REFERÊNCIAS BIBLIOGRÁFICAS}

ALMEIDA M. A. B., GUTIERREZ, G. L., MARQUES R. Qualidade de vida: definições e conceitos. In: Almeida MAB, Gutierrez GL, Marques R, organizadores. Qualidade de vida: definição, conceitos e interfaces com outras áreas de pesquisa. São Paulo: Escola de Artes, Ciências e Humanidades, Universidade de São Paulo; 2012.

ARAUJO FILHO, A. C. A. et al. Perfil epidemiológico do Diabetes Mellitus em um estado do nordeste brasileiro. Rev. pesqui. cuid. fundam. 2017.

AGRANIONIH, N. T. A Teoria da Transposição Didática e o Processo e Didatização dos Conteúdos Matemáticos. Educere - Revista da Educação Unipar. v. 1, n. 1 (2001)

BRASIL, VIGITEL. Hábitos dos brasileiros impactam no crescimento da obesidade e aumenta prevalência de diabetes e hipertensão. 2016.CRUZ, Rielly Maria et al. Assistência integral a diabéticos na atenção básica: análise do $1^{\circ}$ ciclo do PMAQ no município de João Pessoa-PB. Archives of Health Investigation, v. 6, n. 10, 2017.

BRASIL, Ministério da Saúde. A vigilância, o controle e a prevenção de doenças crônicas não transmissíveis: DCNT no contexto do Sistema Único de Saúde brasileiro/ Ministério da Saúde - Brasília - organização Pan-americana de Saúde. 2005.

BARBIER,J.Savoirsthéoriquesetsavoirsd'action.Paris:PressesUniversitairesdeFrance, 1996.

BORGES, C. Saberes docentes: diferentes tipologias e classificações de um campo de pesquisa. Revista Educação \& Sociedade, XXII n. 74, 59-76. Abril / 2009.

BRASIL, Instituto Brasileiro de Geografia e Estatística (IBGE). Disponível em: $<$ https://cidades.ibge.gov.br/brasil/ma/coelho-neto> Acesso em 15 fev. 2017.

COSTA, A. S. \& MARQUEZI, M. L. Implicações do jejum e restrição de carboidratos sobre a oxidação de substratos. Revista Mackenzie de educação Física e Esporte, v. 7, n. 1, 2008.

CRUZ, R. M. et al. Assistência integral a diabéticos na atenção básica: análise do $1^{\circ}$ ciclo do PMAQ no município de João Pessoa-PB. Archives of Health Investigation, v. 6, n. $10,2017$.

CARLSEN, W. (1991). Effects of New Biology Teachers' Subject-Matter Knowledge on Curricular Planning. Science Education, 75(6), 631-647.

Rev. Caminhos da Educação: diálogos, culturas e diversidades, Teresina, v. 1, n. 3, p. 149-165, set./dez. 2019 
Educação alimentar: promoção da saúde através da didatização nutricional associada à atividade fisica para portadores de diabetes mellitus, hipertensos e obesos

DEVELAY,M.Peut-onformerdesenseignants?Paris:E.S.F. 1994.

DOMINGUINI, Lucas. A transposição didática como intermediadora do conhecimento científico e do conhecimento escolar. Revista Eletrônica de Ciências da Educação, Campo Largo, v. 7, n. 2, nov. 2008.

FILHA, C. et al. Avaliação do controle de hipertensão e diabetes na Atenção Básica: perspectiva de profissionais e usuários. Saúde em Debate, v. 38, p. 265-278, 2014.

FILHO, D. R. et al. Tratado de Nutrologia. 2018: Editora Manole Ltda, 2018.

GAUTHIER, C. (Ed.), DESBIENS, J.-F., MALO,A., MARTINEAU, S.\&SIMARD, D.(1997). Pour une théorie dela pédagogie. Recherches contemporaines surles avoir de senseignants.Paris Bruxelles:DeBoeckUniversité,Perspectivesenéducation.

GOMES, R. MENDES, A. N. N. B. TRANSPOSIÇÃO DIDÁTICA E DIDATIZAÇÃO NO CAMPO DA EDUCAÇÃO AMBIENTAL E OS MATERIAIS DIDÁTICOS IMPRESSOS. letras Macapá, v. 7, n. 1, $1^{\circ}$ semestre, 2017

MÈDART, J. Nutrição guia prático de medicina. Lisboa: João Costa, 2005.

MENON, D.SANTOS, J. S. dos. Consumo de Proteína por Praticantes de Musculação que Objetivam Hipertrofia Muscular. Rev Bras Med Esporte - Vol. 18, No 1 - Jan/Fev, 2012

MOTTA, M. D. C.et al. Educação em saúde junto a idosos com hipertensão e diabetes: estudo descritivo. Revista Uningá Review, v. 18, n. 2, 2018.

PEREZ, E. A.. Uso do Hiperdia na Atenção Básica. Disponível em: $<$ http://189.28.128.100/dab/docs/eventos/mostra/rd/ok15h_edson_perez_rd.pdf $>$.

Acesso em 10 nov. 2019.

PETERMANN, X. B. et al. Epidemiologia e cuidado à Diabetes Mellitus praticado na Atenção Primária à Saúde: uma revisão narrativa. Saúde (Santa Maria), v. 41, n. 1, p. 49-56, 2015.

SANTOS, G. M. dos; SOUSA, P. V. de L.; BARROS, N. V. dos A. Perfil epidemiológico dos idosos diabéticos cadastrados no programa hiperdia no estado do Piauí, Brasil. Revista de Atenção à Saúde (antiga Rev. Bras. Ciên. Saúde), v. 16, n. 56, p. 48-53, 2018.

SILVA, E. C. et al. Prevalência de hipertensão arterial sistêmica e fatores associados em homens e mulheres residentes em municípios da Amazônia Legal. Revista Brasileira de Epidemiologia, v. 19, p. 38-51, 2016.

Sociedade Brasileira de Diabetes, Brasil. Diretrizes da Sociedade Brasileira de Diabetes (2017-2018). São Paulo; 2017.

Rev. Caminhos da Educação: diálogos, culturas e diversidades, Teresina, v. 1, n. 3, p. 149-165, set./dez. 2019 
SZWARCWALD, C. L. et al. Recomendações e práticas dos comportamentos saudáveis entre indivíduos com diagnóstico de hipertensão arterial e diabetes no Brasil: Pesquisa Nacional de Saúde (PNS), 2013. Revista Brasileira de Epidemiologia, v. 18, p. 132$145,2015$.

WHELTON, P. K.; CAREY, R. M. The 2017 clinicalpracticeguideline for high bloodpressure. Jama, v. 318, n. 21, p. 2073-2074, 2017. 